
internationales

vol. 30 - $n^{\circ} 2$ | 2014

Composer (avec) la frontière. Passages, parcours migratoires et échanges sociaux

\title{
Figés dans le mouvement : périodes et espaces d'attente des migrants mexicains expulsés des États-Unis
}

Suspended in the Movement: Waiting Periods and Spaces for Mexicans Deported from the United States

Suspendidos en el movimiento: periodos y espacios de espera de los migrantes mexicanos deportados de los Estados Unidos

Olga Odgers-Ortiz et Amalia-E. Campos-Delgado

\section{(2) OpenEdition}

Journals

Édition électronique

URL : https://journals.openedition.org/remi/6922

DOI : $10.4000 /$ remi.6922

ISSN : $1777-5418$

Éditeur

Université de Poitiers

Édition imprimée

Date de publication : 1 juin 2014

Pagination : 113-135

ISBN : 979-10-90426-22-1

ISSN : 0765-0752

Référence électronique

Olga Odgers-Ortiz et Amalia-E. Campos-Delgado, « Figés dans le mouvement : périodes et espaces d'attente des migrants mexicains expulsés des États-Unis », Revue européenne des migrations internationales [En ligne], vol. 30 - n² | 2014, mis en ligne le 01 juin 2017, consulté le 15 avril 2022. URL : http://journals.openedition.org/remi/6922 ; DOI : https://doi.org/10.4000/remi.6922 


\title{
Figés dans le mouvement : périodes et espaces d'attente des migrants mexicains expulsés des États-Unis
}

\author{
... Olga Odgers Ortiz ${ }^{1}$ \\ et Amalia E. Campos Delgado²
}

\section{Introduction}

À l'instar d'autres zones frontalières du globe, le Mexique et les États-Unis ont connu des cycles d'intensification et de ralentissement de leurs flux migratoires. On ne peut cependant manquer de constater que leur impact et leurs conséquences ont pris une importance considérable sous l'effet de l'accélération de la mondialisation économique, et notamment en raison de la densification des champs migratoires contemporains (Simon, 2008) résultant du développement de nouvelles formes de communication et de transport.

L'un des traits les plus saillants des processus de mondialisation à l'œuvre depuis le milieu des années 1970 a sans nul doute été l'intensification de la mobilité, simultanée au processus de renforcement du contrôle des frontières. La transnationalisation des marchés du travail et les déplacements de populations victimes de conflits belliqueux ou de catastrophes naturelles ont contribué à l'apparition de circuits de transit qu'empruntent des millions de personnes. Inscrite dans une "envie globale " de mobilité, la migration est désormais un phénomène structurel des sociétés contemporaines (Wihtol de Wenden, 2012). L'augmentation progressive de la vitesse des déplacements et des interactions humaines a fini par constituer de vastes champs migratoires où la circulation de personnes, de produits et de pratiques culturelles met en relation des points d'origine, de transit et d'installation (Simon, 2008).

Le champ migratoire Mexique/États-Unis est emblématique de ce processus, et ce pour plusieurs raisons : l'intensité des interactions existant entre les deux

\footnotetext{
1 Professeur-Chercheur, El Colegio de la Frontera Norte (COLEF), Blvd. Abelardo L. Rodriguez, 2925, Zona del Río, Tijuana, Baja California 22320, Mexique ; odgers@colef. $\mathrm{mx}$

2 El Colegio de la Frontera Norte (COLEF), Blvd. Abelardo L. Rodriguez, 2925, Zona del Río,Tijuana, Baja California 22320, Mexique ; amalia.cd@gmail.com
} 
nations, l'ancienneté du flux migratoire, mais aussi et tout particulièrement la rapidité des déplacements et de leur ampleur.

La structuration du champ migratoire a progressé au cours du XXe siècle, non par le biais d'un flux migratoire constant, mais comme le résultat de l'alternance de périodes d'accélération et de ralentissement plus ou moins prononcées. On observe néanmoins la consolidation d'un certain ralentissement au début du XXle siècle, moment où le système migratoire commence à manifester des signes d'épuisement; la crise économique de 2008 a quant à elle inauguré une étape de nette stagnation (Massey et Durand, 2003 ; Alarcón et al., 2009).

Ce tournant est évident au vu de la diminution du nombre de passages de la frontière dans le sens sud-nord, faisant suite à la réduction du nombre de nouveaux migrants intégrant le marché du travail transnational ; en parallèle, on constate une perte de vitesse du phénomène de la circularité migratoire (Zúñiga et Arroyo, 2006).

\section{Graphique 1 : Nombre de passages annuels à la frontière} entre le Mexique et les États-Unis (1995-2010)

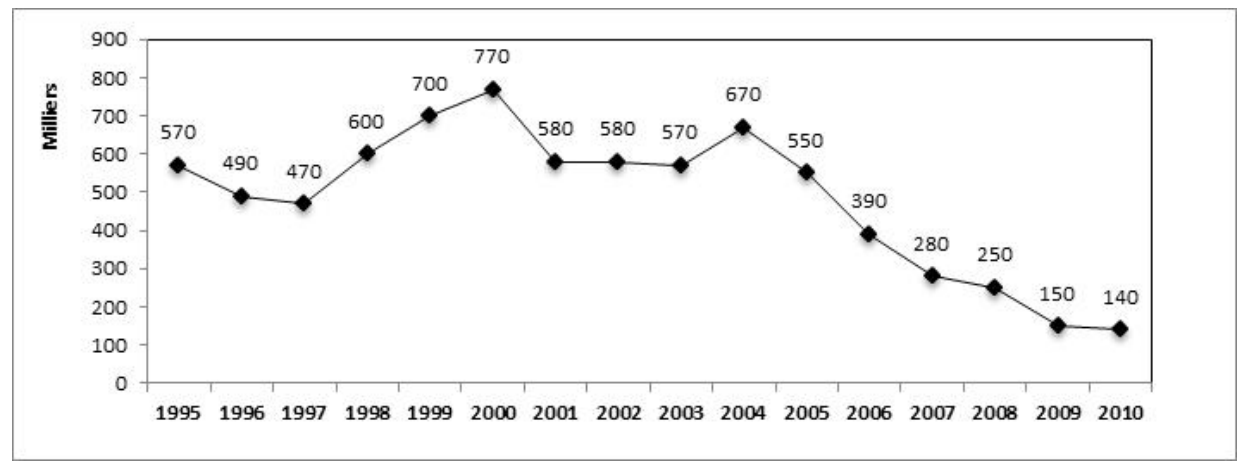

Source : Pew Hispanic Center, estimations calculées sur la base de sources diverses (pour la méthodologie, voir Passel et al., 2012).

Par ailleurs, outre la diminution du nombre de passages annuels, l'augmentation constante des chiffres des retours - volontaires ou forcés - constitue l'un des signes les plus visibles de l'épuisement du système migratoire et de la transformation des modèles de mobilité. L'annonce en 2010 de l'établissement d'un solde migratoire zéro pour le Mexique, n'a fait que confirmer ce qui était déjà une évidence.

L'ampleur des retombées liée à ce changement est plus floue. En effet, si certains aspects comme la chute du nombre de transferts de fonds monétaires peuvent être comptabilisés avec plus ou moins de précision (Canales, 2008), il est plus difficile d'apprécier l'impact de l'épuisement de ce système migratoire sur les relations interpersonnelles qu'entretiennent ceux qui essayent de construire leur vie et celle de leurs enfants dans un contexte migratoire en pleine transition. 
Notre postulat est que la transformation de la nature des flux migratoires entre le Mexique et les États-Unis a entraîné l'apparition de nouveaux espaces où les migrants n'ont d'autre possibilité que celle de " tourner en rond ". II s'agit donc d'espaces définis où des centaines de personnes se trouvent "figées ", dans l'impossibilité d'envisager le retour ou de poursuivre leur chemin, en attente au cœur du mouvement (Musset, 2013).

C'est le cas de ces jeunes nés dans les villes mexicaines transnationalisées et qui ont consacré la majeure partie de leur vie et de leurs rêves à préparer le grand voyage ; ils voient l'un après l'autre leur projet se briser contre le mur érigé à la frontière.

C'est aussi le cas de ceux qui échouent à mi-chemin, à l'intérieur des ÉtatsUnis, puisque le durcissement des contrôles réalisés par le Department of Homeland Security (DHS) rend difficile la circulation de ceux qui risquent l'expulsion. Aquino, par exemple, montre comment les jeunes Indiens, qui ne peuvent pas rejoindre les grandes villes en raison des contrôles migratoires mis en place sur les autoroutes, se retrouvent "emprisonnés dehors", cantonnés dans les emplois précaires qu'offre le secteur de l'agriculture (2012: 27). Le cas le plus significatif reste cependant celui des personnes établies de longue date au nord de la frontière, et qui se voient contraintes au retour après avoir été expulsées. Même si les cas de figure sont divers, nous nous pencherons plus particulièrement dans les pages qui suivent sur la situation de ceux qui se trouvent forcés au « retour " vers un pays qui n'est plus le leur.

Dans cet article, nous présenterons, tout d'abord, très brièvement les caractéristiques du champ migratoire Mexique/États-Unis, afin de montrer comment l'évolution récente des modèles de mobilité a généré des processus sociaux conduisant à la formation de "bulles " qui piègent et retiennent des migrants pourtant inscrits dans une logique de mouvement. Nous décrirons ensuite quelques-uns des effets induits par les transformations de la politique migratoire américaine en matière d'expulsions. Dans un troisième temps, nous présenterons quelques cas significatifs de parcours suivis par les expulsés, dans le but d'établir un modèle des stratégies adoptées par les migrants en fonction de leur possibilité de s'inscrire ou non dans un processus de mobilité (Campos et Odgers, 2013). Nous verrons qu'il existe ainsi différentes manières de se retrouver "figés en plein mouvement ". Enfin, nous présenterons de façon détaillée le cas de ces personnes expulsées, qui se retrouvent dans cet espace frontalier, sans pouvoir rejoindre leur famille au Nord et sans pouvoir retourner à un lieu d'origine déjà oublié. L'analyse de ces cas de figure nous semble importante dans la mesure où ils permettent de contribuer à la réflexion sur la construction d'ores et déjà mondialisée de nouveaux espaces d'attente et de liminalité associés à des processus d'exclusion transnationale et à la " gestion transnationalisée des indésirables " (Agier, 2008).

Le matériel ethnographique que nous présentons provient de trois sources principales. Tout d'abord, nous avons repris des matériaux recueillis lors du travail de terrain réalisé à Los Angeles (Californie) entre juillet 2008 et janvier 2012 dans le cadre du projet Intégration urbaine : facteurs politiques et socio- 
démographiques de l'incorporation des migrants mexicains à Los Angeles ${ }^{3}$. Ensuite, le travail ethnographique mené en 2009-2010 à la frontière Tijuana/San Diego $^{4}$ a également constitué une source précieuse. Enfin, entre mai 2013 et mai 2014, nous avons réalisé plusieurs observations de terrain, mené des entretiens poussés auprès de migrants récemment expulsés et tiré profit de nombreuses conversations informelles dans la ville de Tijuana et plus particulièrement à la Maison du migrant et à la Soupe populaire du Père Chava ${ }^{5}$.

Par ailleurs, afin de recueillir davantage d'éléments concernant la période après l'expulsion nous avons repris contact à l'automne 2013 avec une partie des personnes interviewées à Los Angeles en 2008 et qui avaient déclaré avoir dans leur entourage des personnes expulsées récemment.

Cet article présente les exemples les plus significatifs des processus analysés, mais il est évident que l'ensemble du matériel ethnographique a été d'une grande utilité pour mieux discerner les processus d'expulsion et leurs conséquences ainsi que les stratégies élaborées pour les affronter.

\section{Les aléas de I'histoire}

L'histoire du système migratoire Mexique/États-Unis peut se diviser en périodes alternant accélération et ralentissement des flux, qui correspondent aux niveaux de croissance économique et à la demande de main-d'œuvre, et en périodes de crise accompagnées de vagues d'expulsions des sans-papiers (Durand, 1994 ; Odgers, 2003).

Au début des années 1980, le Service d'immigration et de naturalisation (INS) estime à environ 8 millions le nombre de Mexicains résidant aux États-Unis (Durand, 1998), dont 1300000 réputés sans-papiers (Bustamante, 1997). En 1986, afin de reprendre le contrôle du flux migratoire, est promulguée la loi IRCA (Immigration Reform and Control Act), censée mettre fin à la migration clandestine, en recourant à de nouvelles restrictions et des procédures de régularisation de certains migrants. Cette nouvelle politique d'immigration donne la priorité au regroupement familial pour l'obtention du permis de séjour, modifiant le profil du flux migratoire : les travailleurs sans-papiers qui parviennent à régulariser leur situation grâce à I'IRCA peuvent demander la venue de leur famille. Les régularisations prévues par I'IRCA permettent à de nombreux Mexicains de franchir plus facilement et plus fréquemment la frontière, favorisant ainsi le resserrement des liens entre les émigrants et leurs villages d'origine.

3 La méthodologie et les principales conclusions de ce projet financé par la Fondation BBVA peuvent être consultées dans Alarcón, Escala et Odgers (2012).

4 Anticiper le futur. L'expression d'imaginaires frontaliers dans les projets de vie de jeunes de Tijuana (Mexique) et Tecún Uman, (Guatemala). La méthodologie et une partie des principales conclusions de ce projet peuvent être consultées dans Campos et Odgers (2012).

5 Ces deux espaces créés et administrés par l'Église catholique fonctionnent grâce à l'aide de bénévoles. Les entretiens furent conduits avec l'autorisation des responsables et en vertu du principe du consentement préalable en connaissance de cause. 
La période 1996-2001 se caractérise également par la mise en place de nouvelles mesures législatives restrictives et le développement d'un sentiment latent de xénophobie. Le renforcement de la frontière (durcissement des contrôles et augmentation des droits de passage) marque définitivement cette période. Simultanément, l'absence de mécanismes de régularisation a entraîné le développement d'un modèle de familles " mixtes " dont les membres présentent des statuts migratoires divers, allant de la naturalisation jusqu'à la situation irrégulière, en passant par la résidence permanente obtenue légalement. Les familles comprenant des parents sans-papiers d'enfants possédant la nationalité nord-américaine sont plus nombreuses.

La tragédie des attentats du 11 septembre 2001 a ouvert une nouvelle étape : le fait en soi ne modifie pas les flux migratoires, mais on observe qu'un changement s'opère dans le discours officiel, dans les mesures politiques adoptées, ainsi qu'au sein même de l'opinion publique. En effet, le contrôle des frontières est de plus en plus souvent associé aux questions de défense et de sécurité nationale. Cette dernière étape, qui correspond toujours au contexte actuel, sera abordée de façon détaillée infra.

\section{Les nouveaux processus d'expulsion}

Les attentats du 11 septembre 2001 ont été récupérés par le discours officiel et par les médias et présentés comme le résultat de la permissivité du système de contrôle migratoire et frontalier. Même si les terroristes sont entrés légalement sur le territoire, munis d'un visa, et par des ports d'entrée dûment autorisés, la rhétorique officielle a insisté sur la vulnérabilité des frontières terrestres, ainsi que sur les défaillances des mécanismes de contrôle migratoire et douanier (Hernández, $2008: 8$ ).

Ainsi, à partir de ce moment-là, la restructuration et le renforcement des agences et des dispositifs de contrôle sont devenus prioritaires. Le Department of Homeland Security, un an après sa création, institue en 2003 deux agences dont l'objectif $n^{\prime}$ est autre que la mise en place de la législation en matière migratoire sur le territoire des États-Unis : I'agence Customs and Border Protection (CBP) et I'agence Immigration and Customs Enforcement (ICE) (Verea, 2003 : 191).

La CBP a pour mission prioritaire " d'empêcher l'entrée de terroristes et d'armes terroristes aux États-Unis et de garantir la sécurité de cette nation dans ses frontières et ses ports d'entrée ". Cette agence est chargée du travail quotidien de régulation du flux de personnes aux frontières (Meissner et al., 2013 : 29). De son côté, I'ICE a pour mission de promouvoir la sécurité nationale à travers l'exécution pénale et civile des lois fédérales régissant le contrôle des frontières, des douanes, du commerce et de l'immigration. L'ICE soutient que ses activités contribuent à augmenter la sécurité des États-Unis, dans la mesure où elle se doit d'identifier, arrêter ou expulser les étrangers susceptibles de représenter un danger pour la sécurité nationale ou un risque pour la sécurité publique, ainsi que ceux qui entrent aux États-Unis illégalement ou qui minent par tout autre moyen l'intégrité des lois sur l'immigration et les efforts associés 
de contrôle frontalier ${ }^{6}$. Cette restructuration s'accompagne d'une augmentation considérable des fonds destinés aux agences nouvellement créées. Ils sont multipliés par deux entre 2001 et $2003^{7}$, la CBP s'arrogeant la part du lion ${ }^{8}$. II s'agit du point de départ d'une augmentation ininterrompue d'un budget qui s'élevait en 2009 à près de 18 milliards de dollars (dont $65 \%$ pour la CBP) ${ }^{9}$.

Il est remarquable qu'au cours des années suivantes, en pleine crise économique, les réductions budgétaires qui ont frappé d'autres départements du DHS (santé, transport, sécurité, administration, détection nucléaire, etc.) n'ont jamais concerné les deux Bureaux en charge de la protection frontalière et du contrôle migratoire.

Même si le DHS a été établi à des fins de protection face aux menaces terroristes, son effet le plus évident a été de semer la terreur parmi les migrants sans-papiers originaires du Mexique et de l'Amérique centrale, dans la mesure où il a institué des actions diverses permettant l'arrestation des sans-papiers, y compris loin des lignes frontalières, dans n'importe quel endroit de l'espace public (Fernández et Massey, 2007 : 1098). Les lieux de résidence et de travail, les rues et même les écoles sont devenus des espaces où I'ICE est susceptible de mener des rafles contre les immigrés. ${ }^{10}$.

Le DHS soutient qu'il concentre l'essentiel de ses efforts sur les mécanismes visant à faciliter la migration légale, ainsi que sur l'identification et l'expulsion des « étrangers criminels, les transgresseurs récidivistes des dispositions migratoires et les fugitifs de l'immigration "(DHS, 2013 : 15). Par ailleurs, il affirme travailler en coordination avec le Département de Justice, afin de donner priorité à l'expulsion de migrants ayant un profil criminel et favoriser le recours à des procédures d'expulsions qui visent à réduire le temps de détention des migrants sans-papiers (DHS, 2012 : 10). Cependant, comme nous le verrons, I'un des résultats les plus évidents de cette restructuration a été l'expulsion d'immigrants sans casier judiciaire et pleinement établis aux États-Unis.

Pour appréhender le revirement des tendances en matière d'expulsion et ses conséquences, il est nécessaire d'en distinguer deux modalités. Il y a, d'une part, le cas des returns (retours), c'est-à-dire les sorties du territoire sans ordre judiciaire préalable : ils concernent généralement des Mexicains arrêtés par la patrouille frontalière au moment de la traversée et qui sont "rendus " au Mexique de manière accélérée. Dans la mesure où la quantité de traversées transfrontalières diminue (voir Graphique 1), les arrestations aux frontières diminuent aussi.

6 Voir http://www.ice.gov/about/offices/enforcement-removal-operations/

7 4,9 milliards de dollars en 2001 contre 9,28 milliards en 2003.

86,55 milliards pour la CBP contre 2,73 milliards pour I'ICE.

911,95 pour la CBP et 5,95 pour I'ICE.

10 II convient de souligner les différences importantes qui caractérisent les dispositifs mis en place par I'ICE au sein de chaque État, voire au sein des différentes villes d'un même comté (Alarcón, Escala et Odgers, 2012), en dépit du fait qu'elle constitue une entité fédérale. 
D'autre part, il y a les removals (renvois). Ils désignent des sorties rendues obligatoires par un ordre judiciaire d'expulsion. Ils sont classifiés en renvois " criminels " et " non criminels ". À la différence des returns, les removals concernent des personnes établies aux États-Unis, et qui se voient donc arrachées à leur environnement - famille, emploi, réseaux sociaux - pour " retourner » dans un endroit qui n'est plus leur espace de vie. Le graphique 2 permet d'observer que la diminution globale du nombre de personnes expulsées est uniquement liée à la diminution des arrestations réalisées à la frontière. Simultanément, on observe une augmentation constante des expulsions concernant des personnes déjà installées aux États-Unis.

Graphique 2 : Migrants mexicains : removals et Returns (2001-2011) ; chiffres officiels du Department of Homeland Security

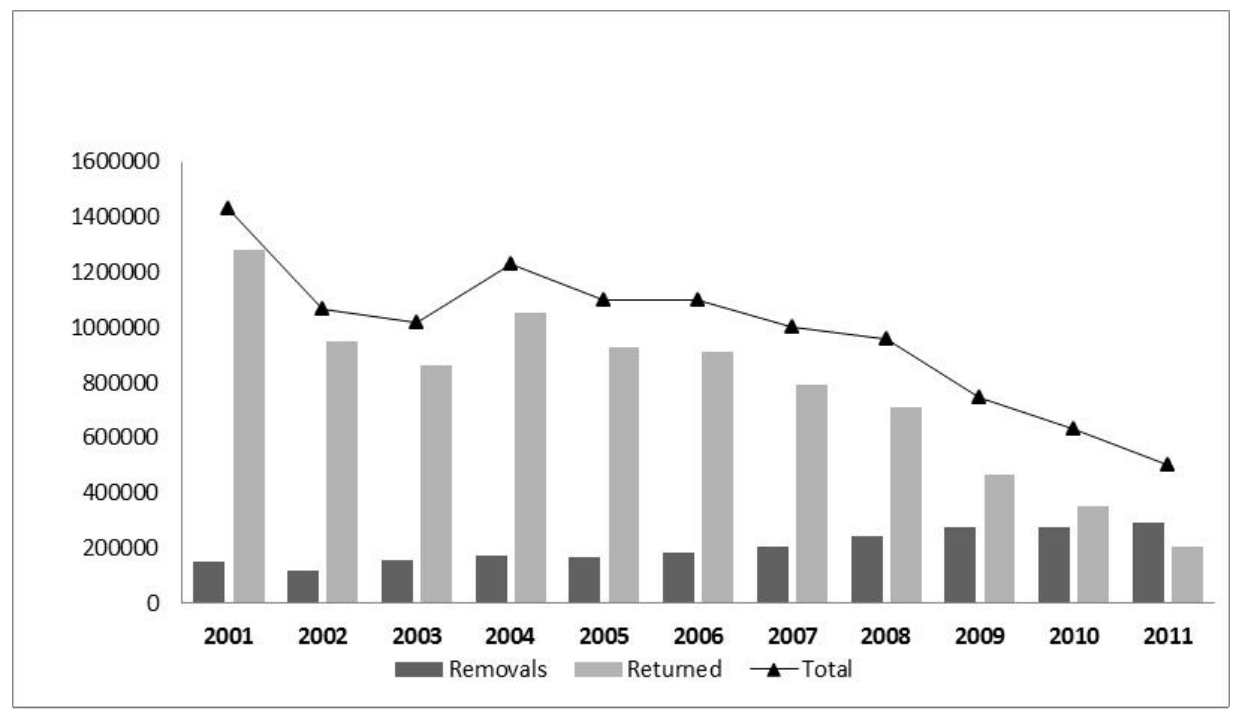

Source : DHS, 2012.

Par ailleurs, dans le groupe des removals, le nombre de migrants non criminels renvoyés augmente régulièrement jusqu'en 2009 (voir Graphique 3). Cette année-là, I'augmentation apparente des " criminels " renvoyés n'est pas due à un changement du profil des expulsés, mais plutôt à une définition élargie du terme "criminel $"^{11}$. Environ $68 \%$ des fautes commises par les criminal returns sont de caractère non violent ; il s'agit, en général d'infractions au code de la route ou d'actes de récidive du franchissement illégal de la frontière (Padilla, 2012 : 219).

11 À partir de 2005, avec le début de l'Opération Streamline, le premier franchissement irrégulier de la frontière est typifié comme un simple délit mineur (misdemanor), donnant lieu à une peine maximale de six mois d'emprisonnement. Cependant, si une personne est de nouveau arrêtée, la récidive sera considérée comme un délit fédéral aggravé (felony) et la peine d'emprisonnement encourue pourra aller de deux à vingt ans (Lydgate, $2010: 3$ ). 
Graphique 3 : Migrants mexicains removed par profil (2002-2011) ; chiffres officiels du Department of Homeland Security

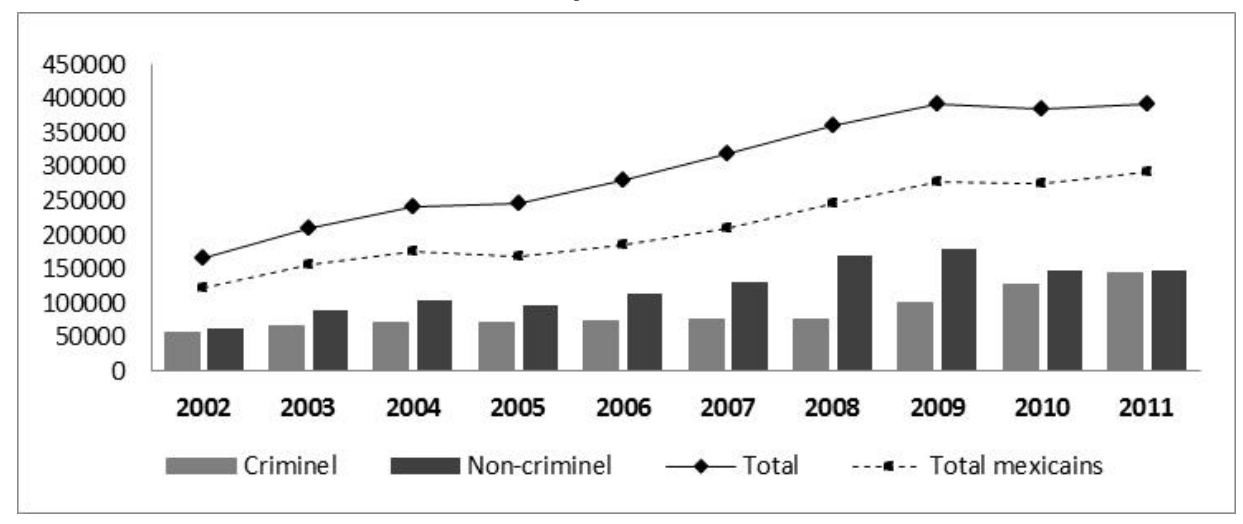

Source : DHS, 2011.

Selon les données de l'enquête menée sur la question migratoire au niveau de la frontière nord du Mexique (EMIF-Norte ${ }^{12}$ ), les Mexicains qui arrivent sur le territoire mexicain après avoir été expulsés par les autorités migratoires des États-Unis sont généralement des hommes, chefs de famille, ayant suivi au moins en partie des études dans le cycle secondaire et dont l'âge moyen est d'environ trente ans. On constate l'augmentation récente des expulsions visant des personnes résidant aux États-Unis depuis plus de trois ans (EMIF-Norte, 2011).

Les données de l'EMIF montrent bien que les migrants ayant fait l'objet d'un renvoi proviennent principalement des états frontaliers (Californie, Arizona et Texas ; EMIF-Norte, 2011), tandis que les principaux points d'expulsion sont situés dans les villes de Mexicali, Tijuana, Ciudad Juárez, Nogales et Nuevo Laredo. Tijuana concentre le flux le plus important de migrants reconduits à la frontière. D'ailleurs, en 2010, 40 \% des expulsés interviewés déclaraient avoir traversé la frontière vers le Nord à partir de cette même zone (Berumen, Ramos et Ureta, $2011: 5)$ alors que seulement $4 \%$ d'entre eux sont originaires de la région (EMIF-Norte, 2011).

Un autre élément significatif du nouveau profil des migrants expulsés concerne la séparation de familles dont les membres possèdent des statuts migratoires mixtes. Rien que pour la période 2004-2010, le nombre des migrants mexicains arrêtés puis expulsés seuls alors qu'ils étaient accompagnés de membres de leur famille est passé de 18714 à 52835 (Martínez, 2012).

12 L'enquête menée sur la question migratoire au niveau de la frontière nord du Mexique (EMIF-Norte) a été lancée en 1993 afin de déterminer la magnitude et les caractéristiques des flux de main-d'œuvre entre le Mexique et les Etats-Unis. Les estimations sont fondées sur un travail d'observation constant et prolongé au sein de treize municipalités situées sur la frontière septentrionale. On peut observer quatre flux indépendants : originaire du Sud, originaire des États-Unis, originaire de la frontière nord et le dernier flux concerne des personnes "rendues " par les autorités américaines. 
Graphique 4 : Ancienneté de la résidence aux États-Unis avant expulsion (\%)

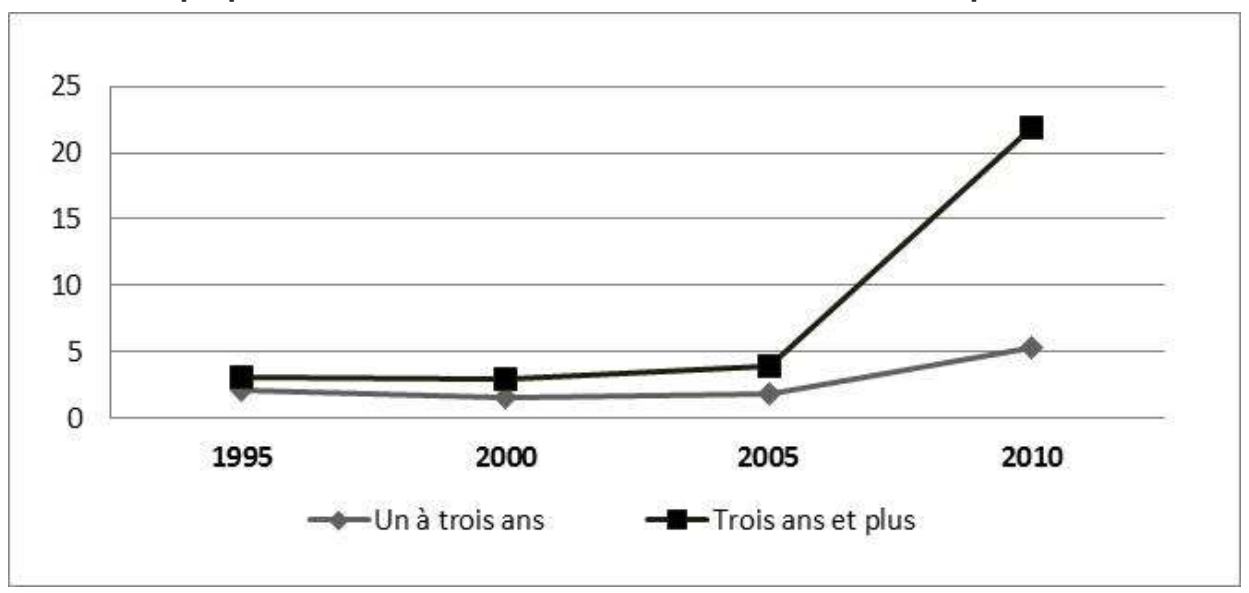

Source : Données de l'enquête EMIF-Norte ${ }^{13}$.

Un cas que l'on retrouve fréquemment dans la composition des familles mixtes est celui des parents migrants en situation irrégulière qui ont des enfants possédant la nationalité états-unienne. Dans ces cas, même si les membres de la famille sont ensemble au moment de l'arrestation, seuls les parents sont expulsés, les enfants devant quant à eux rester aux États-Unis. On estime que 5100 enfants de parents expulsés se trouvent actuellement dans le Foster Care System. Si cette tendance se maintient durant les cinq prochaines années, au moins 15000 enfants supplémentaires se retrouveront dans cette situation (Wessler et al., 2011 : 23).

Graphique 5 : Migrants arrêtés en compagnie de leur famille et expulsés seuls

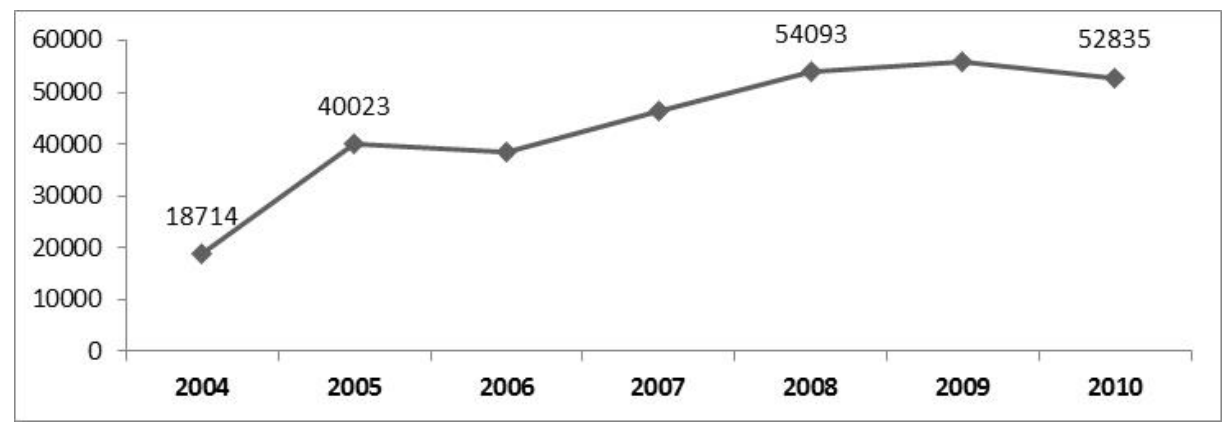

Source : Données extraites de l'enquête EMIF-Norte et citées dans Martínez, 2012.

13 Données extraites par Berumen, Ramos et Ureta (2011 : 8). La méthodologie employée peut être consultée sur http://www.colef.net/emif/diseniometodologico.php 
Ces chiffres permettent d'entrevoir le dilemme des personnes expulsées dont les enfants et/ou le conjoint doivent demeurer sur le territoire nord-américain, alors qu'elles se trouvent contraintes, soit de tenter une réinsertion dans la vie socio-économique du Mexique, soit de tenter de retraverser la frontière pour rejoindre leur famille, courant le risque d'être arrêtées et jugées. Dans cette logique, pour les migrants, la portée temporelle de leur "projet de vie " se trouve donc réduite aux actions immédiates et renvoie à une construction purement émotive du passé et du futur, dont les motivations et les idéaux dictent leurs actions dans le présent (Trommsdorff, 1983 : 396). Autrement dit, pour un bon nombre d'expulsés - notamment ceux ayant laissé derrière eux famille, patrimoine et trajectoires professionnelles -, la construction d'un projet de vie alternatif sur le sol national n'est pas viable ; les actions et les stratégies mises en place seront donc intrinsèquement conjoncturelles, ne visant qu'à faire face à l'expulsion. Comme nous le verrons infra avec le matériel ethnographique présenté, cette situation n'offre aucune échappatoire claire - que ce soit en termes psychologiques, sociaux ou économiques -, alors qu'il est indispensable de pouvoir sortir de cette "bulle " si I'on souhaite parvenir à construire un projet de vie alternatif à celui que ces expulsés avaient forgé aux États-Unis.

\section{Que font les expulsés?}

Qu'il s'agisse d'un retour "volontaire ", quand elles sont arrêtées lors de leur tentative de franchissement de la frontière, ou d'une procédure d'expulsion faisant suite à un procès, les personnes expulsées des États-Unis sont reconduites à la frontière afin qu'elles quittent le territoire à pied, généralement à travers les villes deTijuana ou Ciudad Juárez. Elles ne sont renvoyées par avion à Mexico ${ }^{14}$ que dans des cas exceptionnels.

Dans la plupart des cas, les expulsés se retrouvent donc dans des espaces urbains où ils n'avaient jamais mis les pieds auparavant ou dans lesquels ils avaient séjourné brièvement durant leur transit vers le Nord. Ceux qui ont été expulsés après avoir purgé une peine dans les prisons états-uniennes (pour des périodes pouvant aller de quelques heures à plusieurs années) ne possèdent d'autres biens que ceux qu'ils portaient au moment de leur arrestation.

Lorsqu'ils arrivent au Mexique et pénètrent sur le territoire, les fonctionnaires de I'Instituto Nacional de Migración (INM) les contactent conformément au protocole prévu par le Programme de rapatriement, qui consiste à leur délivrer une attestation d'expulsion, leur fournir de l'eau et de la nourriture pour subvenir à leurs besoins immédiats, leur proposer un soutien médical ou psychologique

14 Le gouvernement fédéral mexicain a mis en place deux programmes à destination de la population expulsée depuis le territoire états-unien, le Programa de Repatriación Voluntaria al Interior (Programme de rapatriement volontaire vers l'intérieur) et le Programa de Repatriación Humana (Programme de rapatriement humain). Le rapatriement volontaire vers l'intérieur du pays a été une initiative lancée en 2006, afin que les migrants originaires du sud et du centre du pays et arrêtés dans les secteurs de Yuma et Tucson puissent revenir par voie aérienne à I'aéroport de Mexico. En 2011, 8893 personnes sont retournées au Mexique selon cette modalité. Le Programme de rapatriement humain, développé dans neuf villes frontalières depuis 2007, a pour objectif de répondre aux nécessités de base des migrants au moment de leur arrivée sur le territoire national. 
d'urgence lorsque cela est jugé nécessaire et, finalement, les conduire, s'ils en font la demande, soit vers un foyer d'accueil temporaire, soit vers l'aéroport ou la gare routière, lorsqu'ils ont la chance de pouvoir acheter un titre de transport et qu'ils ont un lieu de destination (INM, 2014) ${ }^{15}$. II faut préciser que les foyers d'accueil établis dans les villes frontalières ne sont pas gérés par l'État, mais par des ONG locales ou transfrontalières. Le foyer catholique la Casa del Migrante constitue sans aucun doute le foyer le plus connu et le plus actif de la région.

D'un point de vue pratique, à son arrivée sur le territoire mexicain, le premier défi du migrant consiste à dresser un bilan des ressources dont il dispose, afin de pouvoir définir les actions immédiates à entreprendre. II ne pourra commencer à élaborer une stratégie à court et moyen terme qu'après avoir réussi à résoudre les problèmes les plus urgents ; I'accès à des sources de financement ainsi qu'à des réseaux sociaux étendus est ici fondamental (voir infra).

\section{Retourner dans le Nord coûte que coûte}

Dans certains cas, malgré le durcissement du contrôle frontalier et de la législation qui condamne de plus en plus sévèrement le séjour en situation illégale, le retour aux États-Unis est vu comme la seule alternative. Cependant, les difficultés, les coûts et les risques encourus pour y parvenir ne cessent d'augmenter.

Ainsi, lorsqu'Adelaida ${ }^{16}$, la sœur d'Alicia, a été expulsée à I'âge de dix-neuf ans, c'est un large réseau familial qui a dû se mobiliser pour réunir les ressources nécessaires à la traversée sans papiers de la frontière ${ }^{17}$. Elle fait partie de ce que I'on appelle la " nouvelle migration " vers les États-Unis. Postérieure à l'année 2000, elle compte une grande majorité de migrants sans-papiers. La famille d'Adelaida vient de l'État de Veracruz qui compte nombre de migrants qui se caractérisent par leur origine urbaine et par leurs niveaux élevés de scolarité ; ils n'ont cependant que rarement l'occasion de décrocher des emplois qualifiés en raison de leur statut de sans-papiers.

En raison de leur situation socioéconomique quelque peu privilégiée, certains membres de la famille d'Adelaida ont d'abord franchi la frontière en toute légalité, en qualité de touristes, et sont devenus des "sans-papiers " à l'expiration de leur visa. Parmi leurs compatriotes, on trouve divers professionnels venus tenter leur chance de l'autre côté de la frontière après avoir souffert

15 Officiellement, I'INM informe et oriente les rapatriés sur les divers soutiens dont ils peuvent bénéficier au Mexique; il les met en relation avec le consulat mexicain lorsqu'ils souhaitent déposer une plainte contre l'autorité migratoire étrangère et les aide à passer des appels téléphoniques nationaux ou internationaux pour qu'ils entrent en contact avec des membres de leur famille ou toute autre personne de confiance (INM, 2011). Les entretiens réalisés auprès de migrants expulsés permettent de constater que ce n'est pas toujours le cas.

16 Afin de préserver l'anonymat des répondants, tous les prénoms employés sont des pseudonymes.

17 Le premier entretien avec la famille d'Adelaida avait eu lieu en 2008 à Los Angeles juste après qu'Adelaida fût expulsée. Par la suite, en 2013, Alicia, la sœur, fut de nouveau interviewée. Ces deux contacts nous ont permis de recueillir le point de vue des membres de la famille alors qu'ils élaboraient leur stratégie pour le retour, ainsi que les commentaires a posteriori d'Alicia. Nous n'avons pas interviewé Adelaida et nous présentons ici les opinions et les stratégies familiales mises en place pour organiser son retour aux États-Unis. 
des crises économiques à répétition qui ont frappé le Mexique au cours des dernières décennies du XXe siècle. Sa sœur par exemple, pourtant titulaire d'un diplôme universitaire, travaille comme femme de chambre dans un hôtel de la région de Los Angeles.

Arrivée aux États-Unis avant l'âge de trois ans en compagnie de ses parents, Adelaida n'avait jamais pensé devoir retourner au Mexique. Elle a fait toute sa scolarité en anglais et s'exprime difficilement en espagnol. Elle n'a aucun souvenir de son lieu de naissance et plus aucun membre de sa famille ne réside aujourd'hui dans son village natal.

Son second voyage sera différent du premier, plus dangereux et plus coûteux : les passeurs (polleros), qui appartenaient traditionnellement aux communautés d'origine des migrants, ont été progressivement remplacés par les mafias locales liées au trafic de drogues et à la traite des personnes. De l'ordre de 6000 dollars si Adelaida accepte de marcher plusieurs jours dans le désert, ou plus encore si elle contacte les passeurs " haut de gamme ", qui fournissent de faux documents migratoires ou qui contrôlent les rares points de passage à moindre risque. De plus, si la jeune fille venait à être arrêtée de nouveau, le franchissement irrégulier et récidiviste de la frontière entrerait dans la catégorie " aggravated felony ", raison pour laquelle, au lieu de bénéficier d'un retour rapide, elle pourrait être condamnée à purger une peine d'emprisonnement avant d'être expulsée.

Malgré tout, la famille considère qu'Adelaida est encore trop jeune pour commencer une nouvelle vie dans un lieu qui lui est inconnu. Ses parents ont donc fait appel au soutien de la communauté mexicaine, qui a mis à disposition ses maigres économies et mobilisé ses contacts pour permettre à la jeune fille de revenir. Si la traversée de la frontière aboutit, Adelaida devra se montrer extrêmement prudente, une simple infraction au code de la route (misdemanor) pourrait avoir des conséquences désastreuses. Pour sa part, la famille devra économiser pendant plusieurs années afin de rembourser la dette contractée.

\section{Le retour à l'origine : le "rapatrié "}

Dans la plupart des cas, les rapatriés ne disposent pas des ressources qui ont permis à Adelaida de revenir aux États-Unis. Ainsi, face à l'impossibilité d'un retour immédiat dans le Nord, ils doivent aménager leur déplacement vers la communauté d'origine, ne serait-ce que de manière provisoire.

Même si les statistiques disponibles (EMIF-Norte, 2011) indiquent que la plupart des expulsés déclarent avoir l'intention de franchir à nouveau la frontière vers les États-Unis, le renforcement des contrôles et l'augmentation des frais du passage irrégulier rendent cet objectif difficile à atteindre.

C'est pourquoi le retour provisoire vers le lieu d'origine semble être l'alternative la plus courante pour ceux qui disposent de ressources pour payer le déplacement, qui ont gardé un foyer au pays natal - soit la maison des parents, soit celle d'un frère ou d'une sœur ou bien celle de leur propre famille - et qui ont des liens affectifs offrant un refuge face à l'adversité. 
C'est le cas d'Arturo, originaire lui aussi du Veracruz, qui a été arrêté à Los Angeles lorsqu'il rentrait chez lui après le travail ${ }^{18}$. Après avoir passé un peu plus de vingt-quatre heures en détention, Arturo arrive àTijuana au crépuscule. II passe cette première nuit à la Casa del Migrante (Maison du migrant), aux côtés d'une cinquantaine d'hommes récemment expulsés comme lui. Grâce à une connaissance qui habite àTijuana, la cousine d'Arturo réussit à lui faire parvenir depuis Los Angeles l'argent nécessaire pour l'achat d'un billet d'autobus à destination de Veracruz. Arturo décide donc de retourner chez ses parents, tout en sachant qu'il lui sera impossible d'obtenir un emploi lui permettant de retrouver son indépendance économique. Même si Arturo avait commencé à construire une vie stable à Los Angeles, des liens affectifs le rattachent encore à son village. Il pense à franchir à nouveau la frontière parce qu'au village " il n'y a pas d'avenir "; son séjour au Veracruz ne sera donc qu'un temps d'attente, une pause avant de retenter le voyage.

Arturo a rejoint les rangs des "rapatriés ", qui devront s'adapter à une réalité qui les avait poussés à partir alors qu'il n'existe aucun programme spécifique visant à faciliter la réinsertion économique ou sociale des expulsés. C'est pourquoi, même si le terme "rapatrié " utilisé dans le discours officiel prétend promouvoir l'image d'un retour vers une patrie généreuse qui attend le migrant, en réalité les expulsés sont accueillis par un État qui considère leur présence comme un mal inévitable, tandis que les instances gouvernementales, fédérales, étatiques ou locales, ne prennent aucune initiative en faveur de leur réintégration. Les réseaux familiaux - lorsqu'ils existent encore - constituent les seules ressources sur lesquelles peuvent compter les expulsés pour leur réinsertion sociale et économique (Padilla, 2012 : 212).

\section{La frontière comme dans une " bulle »}

Malgré la gravité de leur situation, Arturo et Adelaida s'inscrivent dans des trajectoires où le mouvement reste encore possible. Les liens affectifs, les réseaux sociaux et les ressources financières dont ils disposent, leur permettent, à un coût certes élevé, d'accéder à la mobilité. Mais tous les expulsés n'ont pas ce privilège.

Nous aborderons à présent le cas de ceux qui se retrouvent " attrapés " dans la région frontalière, ne pouvant plus se déplacer ni vers le Nord, ni vers le Sud.

\section{L'impossibilité du retour}

Quand on sait que la majorité des expulsés vient d'autres régions du pays, on peut mieux comprendre les difficultés du retour. Par exemple, pour ceux qui sont originaires du Oaxaca, cela représente concrètement un voyage par voie terrestre d'une durée de cinquante heures environ depuis la ville de Tijuana, à un coût d'environ 200 dollars par personne ${ }^{19}$.

18 Nous avons interviewé la cousine d'Arturo en 2008 à Los Angeles. Après I'expulsion en 2009, elle lui a fait parvenir des nouvelles par notre intermédiaire. Nous avons pu ainsi interviewer Arturo à Tijuana et nous avons pu suivre I'évolution de sa situation jusqu'en 2013, lorsque nous avons à nouveau rencontré sa cousine.

19 Le Programa de Repatriación Humana prévoit une aide aux démarches pour obtenir une réduction allant de $25 \%$ à $50 \%$ du prix total du billet (INM, 2012 : 18). 
Même si les organisations de ressortissants d'un même village ou d'une même région (Organizaciones de oriundos) ont une expérience de plusieurs décennies dans la construction de réseaux de soutien aux migrants (Alarcón et Escala, 2007), le retour dans les communautés d'origine est complexe, en raison notamment des mauvaises conditions socioéconomiques et du fait aussi que ces organisations soutiennent davantage ceux qui arrivent à destination aux États-Unis que ceux qui sont amenés à retourner dans leurs localités de départ.

Le stigmate de l'échec est également un facteur qui peut peser sur la décision du retour (Brotherton et Barrios, 2009). Le Mexique a construit pendant plus d'un demi-siècle l'image du migrant qui réussit, qui transfère des fonds monétaires et qui vit le rêve américain ; dès lors, le retour peut être perçu comme un échec personnel.

Pour beaucoup de migrants expulsés, le retour à la communauté d'origine implique non seulement de se retrouver dans un endroit méconnu, même s'il s'agit du pays natal idéalisé, mais aussi d'affronter la réalité de la violence liée au crime organisé qui a gagné du terrain sur une bonne partie du territoire mexicain.

Tel est le cas de Mauricio ${ }^{20}$, cinquante-six ans, originaire de la région de Michoacán, qui avait franchi la frontière en 1978 contre la somme de 500 dollars versés à un guide chargé de le conduire jusqu'à Chicago. Après avoir occupé pendant plusieurs années des emplois de déménageur ou d'ouvrier du bâtiment, il obtient de faux papiers et réussit à décrocher un emploi au sein de l'entreprise Playmobil, emploi qu'il occupe pendant près de dix ans.

En 1980 il épouse une citoyenne états-unienne avec laquelle il a deux enfants puis divorce trois ans plus tard et perd le contact. Bien qu'il n'ait jamais exercé comme travailleur agricole, il achète une attestation d'emploi dans ce secteur pour 1000 dollars et obtient la résidence légale à la faveur du processus d'amnistie Simpson-Rodino. II se remarie avec une Mexicaine sans-papiers qui lui donne deux enfants. Dès lors, il exerce plusieurs métiers, dont ceux de vendeur d'aspirateurs et barman. II commence à abuser de l'alcool alors qu'il travaille dans un bar. Lorsqu'il est accusé de violence domestique, le juge ordonne qu'il suive un programme de désintoxication. Cet antécédent aura par la suite de graves conséquences dans la mesure où, en dépit de son statut légal, Mauricio a été expulsé conformément aux nouvelles réglementations qui prévoient la perte de la résidence en cas de délit grave ou d'accumulation de délits mineurs (misdemanor). Dans son cas, sa femme a déposé une nouvelle plainte pour violence : pour elle, cela ouvrait la possibilité de demander un visa humanitaire; pour lui, cela s'est traduit par l'emprisonnement, la perte de la résidence et l'expulsion. Après son arrestation par la police du Comté de Santa Ana, en Californie, il a été remis à l'ICE et a passé un peu plus d'un an et demi en détention, avant d'être finalement expulsé.

Mauricio est arrivé le 6 janvier 2014 en fin d'après-midi dans un pays qu'il ne connaissait qu'à travers de courtes visites. Ses liens familiaux étaient quasiment

20 Mauricio a été interviewé en 2013, peu après son expulsion, à la Casa del migrante, à Tijuana. 
inexistants des deux côtés de la frontière et il ne disposait ni de ressources économiques ni de réseaux de soutien. Au moment de l'entretien dans I'un des foyers d'accueil destinés aux migrants dans la ville deTijuana, Mauricio constate qu'il n'a aucune possibilité de retourner aux États-Unis, pas plus qu'il n'a de possibilités sérieuses de retourner dans sa communauté d'origine - il n'a pas vu sa famille depuis plus de trente ans - et la violence qui règne dans le Michoacán I'inquiète :

"Il y a beaucoup de problèmes, là-bas, il y a trois cartels, ils kidnappent des gens à tout bout de champ ; celui qui ne paye pas, ils le tuent, ils le dépècent puis éparpillent les différentes parties du corps sous les arcades du centre-ville".

Treize jours après son expulsion, Mauricio évalue sa situation :

" Ils me balancent ici [du côté mexicain de la frontière] et moi, je ne veux pas rester, à cause de la situation telle qu'elle est. Mais si je vais là-bas [dans le Michoacán], la situation est encore pire, avec tous ces cartels [...]. Et puis, si je vais là-bas [aux ÉtatsUnis], on va me mettre en prison ".

Le fait de rester àTijuana n'est dans ces conditions pas un choix. Mauricio est dans l'impossibilité de se déplacer et il reste en attente d'une solution qui, selon toute vraisemblance, $n$ 'arrivera que difficilement.

Tout comme Mauricio, nombre de migrants expulsés s'installent de manière provisoire dans des villes telles que Tijuana sans avoir de possibilités de retourner vers le Nord ou vers le Sud. Ils sont là, attrapés et immobiles, dans un espace qui pourtant se caractérise par sa mobilité.

\section{Le Nord à portée de main}

La " bulle frontalière " n'est pas liée uniquement à l'impossibilité du retour. La présence toute proche, côté nord, de l'entourage des migrants - conjoint(e) et enfants, principalement - retient les expulsés dans la zone frontalière.

Lorsque les membres de leur famille ont la possibilité de traverser légalement la frontière - avec un permis de séjour temporaire, en tant que résidents légaux permanents ou du fait d'être devenus citoyens des États-Unis -, rester sur place permet d'avoir quelques visites. Par contre, pour ceux dont les proches sont sans-papiers, la frontière équivaut à vivre "si près, mais pourtant si loin ". La vie frontalière offre la trompeuse promesse de mettre le Nord à portée ; vivre à la frontière entretient l'espoir de la franchir et de rejoindre les siens.

C'est ce qu'explique Ignacio, Indien mixtèque né à Oaxaca, qui a traversé la frontière pour la première fois en 1985, alors qu'il venait de terminer le collège, dans le but de trouver un emploi qui lui permettrait d'envoyer de l'argent à sa mère veuve et à ses jeunes frères ${ }^{21}$.

Les premières années sont marquées par la pauvreté et les difficultés que connaissent tous les migrants irréguliers, mais aussi par la discrimination dont

21 Ignacio fut interviewé à la Casa del Migrante àTijuana en novembre 2013 quelques jours après son expulsion. 
il souffre en raison de sa couleur de peau de la part même de ses compatriotes. Pourtant, au fil du temps, Ignacio abandonne les emplois les moins bien payés de l'agriculture californienne pour consolider sa position de travailleur semiqualifié dans la culture de la fraise sous serre. En Californie, il se marie à une jeune fille née à Oaxaca et qui est elle aussi en situation irrégulière ; ils auront deux filles. Avec quinze heures de travail par jour, il parvient à économiser suffisamment d'argent pour envisager l'achat d'une maison avec un prêt dont il paierait les mensualités.

Alors qu'il vit depuis plus de vingt ans à Los Angeles et que ses deux filles sont nées aux États-Unis, Ignacio veut régulariser sa situation en payant I'un des multiples avocats qui offrent leurs services aux sans-papiers. Son cas, mal géré - aggravé par sa méconnaissance du système juridique nord-américain - se conclut par une sentence d'expulsion, dont il ne prend connaissance que lors de son arrestation à son domicile.

II n'a pas le temps de prévenir quelqu'un, ni de dire au revoir à sa femme ou à ses filles, ni de contacter un avocat. On l'autorise uniquement à téléphoner au consulat du Mexique, où on lui assure qu'on ne peut rien pour lui.

Deux jours plus tard, alors âgé de quarante-cinq ans, il passe la nuit dans un foyer catholique pour migrants expulsés. Au moment de l'entretien, peu de temps après son expulsion, Ignacio explique qu'il pense rester à Tijuana jusqu'au moment de franchir la frontière.

II ne possède que les vêtements qu'il porte sur lui. Sa femme, sans papiers, ne peut pas traverser la frontière pour aller le voir, mais elle commence à explorer la possibilité d'envoyer leurs filles rendre visite à leur père en compagnie d'un membre de la famille. Le risque est grand, car si son épouse est expulsée elle aussi, leurs enfants se verraient obligées d'entrer dans le système du Foster Care (placement des enfants mineurs). II craint que sa femme ne puisse plus continuer à payer les traites de la maison avec son seul salaire, ce qui reviendrait à la perdre. C'est pour cela que, aussitôt arrivé à Tijuana, il a cherché et trouvé un emploi de maçon pour envoyer à sa famille un peu d'argent qu'il gagne en travaillant douze heures par jour. En ce qui concerne la possibilité de faire venir sa famille à Tijuana, il dit :

"Non, c'est que... à vrai dire, la vie aux États-Unis est un petit peu différente. Là-bas,

il y a plus de possibilités de s'en sortir, alors qu'ici, au Mexique... Sans vouloir offenser personne - c'est mon pays -, je trouve ça vraiment très difficile... Et puis, je voulais aussi que ma femme aille à l'école pour apprendre l'anglais comme il faut et pour qu'elle puisse

faire d'autres choses. Non, je n'ai pas prévu de les faire venir, à vrai dire. Je vais plutôt voir si j'arrive à traverser, moi. Je dis à ma femme que j'aimerais que mes filles étudient aux États-Unis, parce que je sens que pour elles ce serait moins dur qu'au Mexique. Ici, il y a des gens de ma famille qui ont une profession et qui sont au chômage. Au Mexique, on souffre beaucoup... et les femmes, encore plus...".

Le cas d'Ignacio n'est pas atypique. Pour beaucoup d'expulsés, la frontière matérialise la proximité avec leur famille et avec le patrimoine constitué au cours de longues années passées aux États-Unis. Dans leur esprit, la porte de sortie deviendra à un moment donné la porte d'entrée et, en attendant, les possibles retrouvailles avec leurs proches leur permettent d'endurer la situation. Ainsi, les 
villes frontalières deviennent des espaces d'attente, les expulsés y vivent dans l'espoir de poursuivre le rêve américain.

\section{Vivre en marge et en être expulsé}

Les cas précédents montrent bien que seuls les réseaux familiaux et communautaires, lorsqu'ils existent, offrent un fragile contrepoids aux processus d'expulsion. Lorsque ces liens sociaux forts viennent à manquer, cela complique encore davantage tout le processus de réintégration. C'est le cas des migrants expulsés qui vivent désormais dans les canalisations du fleuve deTijuana ${ }^{22}$.

Cet espace, mieux connu sous le nom d'El Bordo (le bord) est occupé par des personnes marginalisées en situation de grande vulnérabilité : des indigents sans abri, des toxicomanes et récemment des migrants qui, après avoir été expulsés, n'ont pas trouvé les moyens de s'installer ailleurs ${ }^{23}$. Par ses caractéristiques topographiques (le lit d'une rivière à sec), il maintient ceux qui y cherchent refuge hors de la vue des habitants de la ville qui préfèrent ignorer leur existence ${ }^{24}$.

Cependant, un événement particulier a fait sortir de l'invisibilité les habitants d'El Bordo. En août 2013, en vue des actions de réparation et de nettoyage de la canalisation, la municipalité décide de chasser tous ceux qui y résident, sans avoir envisagé le relogement. Deux jours après son expulsion, le Campamento para migrantes deportados: El Bordo (Campement pour migrants expulsés : El Bordo) s'installe sur une place publique, avec le soutien de l'organisation de la société civile Ángeles Sin Fronteras (Anges sans frontières) ${ }^{25}$. Quelques semaines suffisent pour que les médias fassent d'eux les nouveaux boucs émissaires de la ville, pour que les autorités locales leur fassent porter la responsabilité de l'augmentation de la violence, pour que les "bons citoyens " réclament sur les réseaux sociaux une aide aux expulsés afin qu'ils soient renvoyés dans leur communauté d'origine pour des " raisons d'hygiène publique ", opérant ainsi l'amalgame entre indigents, toxicomanes et migrants expulsés. Le 23 décembre, le campement est démantelé et ses habitants délogés. De manière prévisible, les sans-abri du campement reviennent s'installer à El Bordo, où leur invisibilité physique va de pair avec leur invisibilité dans les réseaux sociaux, les déclarations politiques et les médias.

22 Le fleuve de Tijuana, 195 kilomètres de longueur, prend sa source dans la Sierra de Juárez, en Basse-Californie (au Mexique) et se jette dans I'océan Pacifique en Californie (aux États-Unis).

23 Même si les chiffres officiels signalent une population d'1 millier de personnes, selon certains organismes de la société civile, la canalisation hébergerait plus de 4000 personnes vivant dans les égouts et dans des abris improvisés.

24 Les études universitaires sur cet espace et sur les conditions sociodémographiques de la population qui y habite sont peu nombreuses (Medrano, 2010 ; Ojeda et al., 2011) ; cet endroit a cependant fait l'objet de reportages sérieux, dont les documentaires réalisés par Arte-TV (2008) ou par la revue VICE (2013).

25 Depuis presque dix ans, en raison de son extrême précarité, la population de la canalisation du fleuve deTijuana a fait l'objet de programmes de prise en charge de la part de différentes ONG - souvent transfrontalières et/ou à caractère religieux -, qui tentent de subvenir aux besoins les plus pressants en matière d'alimentation et de santé (voir http:// archive.is/ASGw0). 
Certes, seul un faible pourcentage des habitants d'El Bordo sont des migrants expulsés, mais cet événement a révélé $d^{\prime}$ 'une part que le discours construit autour de ces migrants montre qu'on refoule les expulsés, à la frontière et d'autre part, il met en évidence les mécanismes d'exclusion auxquels font face les migrants et auxquels s'ajoutent d'autres formes d'exclusion. L'accumulation de tels processus peut atteindre des proportions inouïes au cours de l'expérience migratoire.

C'est le cas de Francisco, cinquante-deux ans, qui déclare vivre dans la rue suite à son expulsion de Californie ${ }^{26}$. Originaire de l'état du Jalisco, il traverse la frontière pour la première fois en 1985 après avoir travaillé comme chauffeur de bus àTijuana pendant deux ans. Même s'il ne disposait pas de papiers officiels, son faux permis de travail lui a permis de trouver des emplois dans les secteurs du bâtiment et du transport terrestre, et de voyager ainsi à travers plusieurs villes américaines. "Je vivais comme un roi ", commente-t-il.

Sa vie a changé en 2009, lorsque son " permis de travail "n'a plus été utilisable après la mise en place d'une nouvelle législation beaucoup plus contraignante pour les employeurs (contrôles, système d'amendes) de travailleurs illégaux ou de sans-papiers. Ce document pourtant lui permettait d'accéder au marché du travail. II a pu jouir ainsi de conditions de vie bien meilleures que celles de ses compatriotes, avec lesquels il a peu à peu pris ses distances. Dès lors, dans l'impossibilité d'obtenir un emploi formel, il est également dépourvu de réseaux sociaux efficaces qui lui faciliteraient l'entrée sur le marché du travail clandestin. Ainsi, en dépit de sa situation économique relativement aisée, il ne peut franchir légalement la frontière.

Pour Francisco, l'absence d'emploi formel rend impossible l'accès aux services de santé pourtant nécessaires pour traiter sa condition de diabétique. En quelques mois, ne pouvant plus payer de loyer, il se retrouve à la rue (dans des parcs et des campements de sans-abris). II sera finalement arrêté pour consommation d'alcool sur la voie publique (open container), infraction qui lui vaudra d'être expulsé peu après. II arrive à Tijuana dans un état physique et mental dégradé. Il est alors victime d'abus côté mexicain :

"Tu peux pas accepter un boulot fixe parce que t'as aucune garantie, ni au travail, ni dans la rue pour ce qui est de la sécurité, et à quoi ça me sert de gagner 1500 pesos par semaine si les flics me prennent tout mon argent..." ".

II vit à El Bordo et de temps en temps, il gagne un peu d'argent en déchargeant des camions pour les grands supermarchés. II garde cependant l'espoir de traverser la frontière en toute légalité et de pouvoir retrouver son style de vie de migrant transnational.

26 Francisco fut interviewé en 2014 à la Soupe populaire catholique de la ville de Tijuana. Cet espace, financé et administré par l'Église catholique se situe à quelques mètres d'El Bordo; on y sert des repas aux personnes sans-abri. 


\section{Conclusion : une gestion transnationale des indésirables?}

Dans cet article, nous avons cherché à montrer comment, au fil de la dernière décennie, la logique et la dynamique des déplacements de la population en situation irrégulière dans le champ migratoire Mexique/États-Unis se sont profondément transformées. D'un côté, la circularité migratoire a diminué pendant la période qui démarre, symboliquement, avec le renforcement de la frontière suite aux attentats du 11 septembre 2001 ; de l'autre, le nombre d'expulsions touchant ceux qui ont construit des réseaux affectifs et économiques dans ce pays est en augmentation. Les données témoignent d'une réduction des arrestations lors du franchissement irrégulier de la frontière ; parallèlement, et de manière progressive, le nombre de reconduites à la frontière d'individus ayant séjourné de longues années dans les centres urbains augmente. Les processus d'expulsion acquièrent un caractère plus dramatique encore lorsqu'ils visent des personnes arrachées à leur lieu de vie aux États-Unis et qui laissent derrière elles des proches pour retourner sous la contrainte dans un pays qui, visiblement, ne veut plus d'eux.

Depuis plus d'un demi-siècle, le Mexique a façonné l'image du migrant qui réussit et cherché le moyen de canaliser les transferts de fonds à des fins productives. Cependant, le XXle siècle s'est ouvert sur le dilemme lié au retour des " absents ", laissant dès lors apparaître une fêlure dans le discours et les pratiques de la " patrie généreuse ".

La réalité que vit cette population est complexe et ne peut être réduite à une logique guidée par les notions d'enracinement, nationalité et citoyenneté. Dans le cas d'Adelaida, le retour vers un pays qu'elle a quitté seize ans auparavant revient à affronter un territoire qui lui est totalement étranger. Pour ses proches, la décision à prendre est claire : le retour à n'importe quel prix.

Par ailleurs, l'itinéraire de retour dans la communauté d'origine peut n'être que le début de nouveaux processus d'exclusion : les migrants ne sont pas attendus dans leurs villages, les envois d'argent sont toujours nécessaires et, de ce fait, ils ne sont pas les bienvenus. Tel est le cas d'Arturo, dont le retour au point de départ implique de retrouver à nouveau les conditions qui l'ont poussé à la migration. Commence alors une période d'attente, qui se caractérise par une réadaptation à une réalité éloignée de celle expérimentée en terre étrangère et le soupçon d'échec, stigmate d'autant plus pesant que l'expulsion est associée à une cause criminelle sans qu'importe la nature réelle de la faute.

Pour d'autres, le retour dans leur communauté d'origine n'est pas envisageable, pas plus que le retour sur le territoire des États-Unis. Dès lors, la seule option consiste à rester dans les villes frontalières, lesquelles deviennent des espaces d'attente. C'est le cas de Mauricio, qui s'est installé à Tijuana.

Ces "bulles frontalières " créent en outre l'illusion de la proximité géographique ; c'est un aspect important pour ceux qui, comme Ignacio, ont dû expérimenter la coupure avec leur famille. Rester dans la ville frontalière lui donne au moins la possibilité, serait-elle épisodique, de revoir ses filles, citoyennes des États-Unis. 
Les Mexicains expulsés des États-Unis sont l'exemple des processus d'exclusion transnationaux parallèles à la création d'espaces de liminalité qui deviennent de nouveaux lieux de vie ou d'attente. Dans l'impossibilité d'obtenir un emploi dans leur pays d'origine, ils s'insèrent dans les marchés transnationaux de l'emploi, dont ils se font expulser au premier signe de ralentissement économique. Une fois de retour, ils ne sont plus les bienvenus, soit parce qu'il n'y a plus personne pour les accueillir, soit parce que ceux qui sont restés ont besoin de l'argent en provenance du Nord pour survivre.

Figés à la frontière, ils sont à la porte de deux États qui ne veulent plus d'eux.

Les processus récents d'expulsion dans le champ migratoire Mexique/ÉtatsUnis doivent être analysés au regard de la création de ces nouveaux espaces et temps d'attente. Cette réalité réactualise le besoin d'intégrer à la réflexion une " nouvelle approche spatiale de l'exclusion sociale " (Mathieu, 1997), dans le but d'expliquer les logiques articulant les champs migratoires, variables dans le temps et propices à insérer les individus dans des flux pour les en refouler ensuite.

\section{Références bibliographiques}

Agier Michel (2008) Gérer les indésirables. Des camps de réfugiés au gouvernement humanitaire, Paris, Flammarion, $349 \mathrm{p}$.

Alarcón Rafael, Cruz Rodolfo, Díaz Bautista Alejandro, González König Gabriel, Izquierdo Antonio, Yrizar Guillermo y Zenteno René (2009) La crisis financiera en Estados Unidos y su impacto en la migración mexicana, Migraciones internacionales, $5(1)$, pp. 193-210.

Alarcón Rafael and Escala Rabadán Luis (2007) Transnational Philanthropy and Organizational Strategies: The Challenge of Mexican Hometown Associations in the United States, in Germán Zárate Hoyos Ed., New Perspectives on Remittances from Mexicans and Central Americans in the United States, Kassel, Kassel University Press, pp. 130-158.

Alarcón Acosta Rafael, Escala Rabadán Luis y Odgers Ortiz Olga (2012) Mudando el hogar al norte. Trayectorias de integración de los inmigrantes mexicanos en Los Angeles, México, El Colef, 412 p.

Aquino Moreschi Alejandra (2012) De la milpa al field: la experiencia migratoria de jóvenes zapatistas en los campos de cultivo californianos, LiminaR, 10 (1), pp. 15-32.

Berumen Sandoval Salvador, Ramos Martínez Luis Felipe y Ureta Hernández Isabel (2011) Migrantes mexicanos aprehendidos y devueltos por Estados Unidos. Estimaciones y características generales, Apuntes sobre Migración, 2, pp. 1-10.

Brotherton David C. and Barrios Luis (2009) Displacement and stigma:The socialpsychological crisis of the deportee, Crime Media Culture, 5 (1), pp. 29-55.

Bustamante Jorge (1997) Características de los migrantes mexicanos en Estados Unidos, in Secretaría de Relaciones Exteriores-Commissionon Immigration Reform, Estudio Binacional México-Estados Unidos sobre Migración, México, 1997.

Campos Delgado Amalia E. and Odgers Ortiz Olga (2012) Crossing the border: mobility as a resource in the Tijuana/San Diego and Tecún Umán/Tapachula regions, Revista Estudios Fronterizos, 13 (26), pp. 9-32. 
Canales Alejandro (2008) Vivir del Norte: remesas, desarrollo y pobreza en México, México, Conapo/Segob, 318 p.

Department of Homeland Security US [DHS] (2004-2014) Budgets-in-Brief Fiscal Year (2003-2014), [en ligne] consulté le 01/01/2014. URL : www.dhs.gov/dhsbudget

Department of Homeland Security US [DHS] (2013) Budget-in-Brief Fiscal Year 2013, [en ligne] consulté le 12/01/2014. URL : http://www.dhs.gov/xlibrary/assets/ mgmt/dhs-budget-in-brief-fy2013.pdf

Department of Homeland Security US [DHS] (2012a) 2011Yearbook of Immigration Statistics, [en ligne] consulté le 01/01/2014. URL : http://www.dhs.gov/sites/ default/files/publications/immigration-statistics/yearbook/2011/ois_yb_2011.pdf

Department of Homeland Security US [DHS] (2012b) Immigration Enforcement Actions: 2011, [en ligne] consulté le 12/01/2014. URL : http://www.dhs.gov/sites/ default/files/publications/immigration-statistics/enforcement_ar_2011.pdf

Department of Justice US [DOJ] (2002) Immigration and Naturalization Service Budget FY 1986-2002, [en ligne] consulté le 12/01/2014. URL : www.justice.gov/ archive/jmd/1975_2002/2002/html/page104-108.htm

Durand Jorge (1998) Política, modelos y patrón migratorios. El trabajo y los trabajadores mexicanos en Estados Unidos, San Luis Potosí, El Colsan, 79 p.

Durand Jorge (1994) Más allá de la línea. Patrones migratorios entre México y Estados Unidos, México, CONACULTA, 353 p.

Encuesta sobre Migración en la Frontera Norte de México [Emif-NorTE] (2011) Serie Histórica 2005-2010, México, El Colegio de la Frontera Norte/Secretaría de Gobernación/Secretaría de Trabajo y Previsión Social/Secretaría de Relaciones Exteriores, $300 \mathrm{p}$.

Fernández Kelly Patricia and Massey Douglas S. (2007) Borders for Whom? The Role of NAFTA in Mexico-U.S. Migration, The Annals of the American Academy of Political and Social Science, 610, pp. 98-118.

Hernández Joseph Daniel (2008) Política migratoria y de control fronterizo de Estados Unidos hacia México y Centroamérica, Revista Enfoques, 7 (8), pp. 193-214.

Instituto Nacional de Migración [INM] (2012) Síntesis estadística, México, Segob.

Le Gouil Gwen, Bodinier Jean-Laurent et Vigna Anne (2008) Mexique : les déportés de la "Tierra de nadie ", ARTE GEIE-Cargo Culte Productions, [en ligne] consulté le 01/11/2013. URL : http://www.arte.tv/fr/arte-reportage/2020926.html

Lydgate Joanna (2010) Assembly-Line Justice: A Review of Operation Streamline, [en ligne] consulté le 01/10/2013. URL : https://www.law.berkeley.edu/files/ Operation_Streamline_Policy_Brief.pdf

Martínez Fabiola (2012) Criterios políticos, en las crecientes deportaciones de mexicanos: INM, [en ligne] consulté le 12/09/2013. URL : http://www.jornada. unam.mx/2012/10/21/politica/004n1 pol\#sthash.DsetSNjB.dpuf

Massey Douglas y Jorge Durand (2003) Clandestinos: migración México-Estados Unidos en los albores del siglo XXI, Zacatecas, Universidad autónoma de Zacatecas, $210 \mathrm{p}$. 
Mathieu Nicole (1997) Pour une nouvelle approche spatiale de l'exclusion sociale, in Strates. Matériaux pour la recherche en sciences sociales, 9, [en ligne]. URL : http://strates.revues.org/612

Medrano Villalobos Gerardo (2010) Comunidades consumidoras de heroína. Los discursos ocultos sobre el placer, Tesis de maestría en Estudios Socioculturales, México, El Colef.

Meissner Doris, Kerwin Donald M., Chishti Muzaffaret and Bergeron Claire (2013) Immigration Enforcement in the United States: The Rise of a Formidable Machinery, Washington DC, Migration Policy Institute, 175 p.

Musset Alain (2013) Santiago y sus migrantes: entre espera y esperanza, in Verónica Correa, Idenilso Bartolotto et Alain Musset Dir., Geografías de la espera. Migrar, habitar y trabajar en la ciudad de Santiago, Chile, 1990-2012, Santiago, Uqbar Editores, pp. 17-27.

Odgers Ortiz Olga (2003) Flux migratoire du Mexique vers les États-Unis : changement et continuité, Problèmes d'Amérique Latine, 50, pp. 59-78.

Ojeda Victoria D., Robertson Angela M., Hiller Sarah P., Lozada Remedios, Cornelius Wayne, Palinkas Lawrence A., Magis Rodriguez Carlos and Strathdee Steffanie A. (2011) A Qualitative View of Drug Use Behaviors of Mexican Male Injection Drug Users Deported from the United States, Journal of Urban Health, $88(1)$, pp. 104-117.

Padilla Delgado Antonio (2012) ¿Repatriado? Una historia de vida y su contexto, in María Dolores París Pombo Ed., Migrantes, desplazados, braceros y deportados. Experiencias migratorias y prácticas políticas, México, El Colef/UACJ/ UAM-Xochimilco, pp. 209-248.

Passel Jeffrey S., Cohn D'Vera and González Barrera Ana (2012) Net Migration from Mexico Falls to Zero-and Perhaps Less, Washington DC, Pew Hispanic Center, [en ligne] consulté le 18/11/2013. URL : http://www.pewhispanic.org/2012/04/23/ net-migration-from-mexico-falls-to-zero-and-perhaps-less/

Simon Gildas (2008) La planète migratoire dans la mondialisation, Paris, Armand Colin, 2008, 255 p.

Trommsdorff Gisela (1983) Future orientation and socialization, International Journal of Psychology, 18 (1-4), pp. 381-406.

Verea Mónica (2003) Migración temporal en América del Norte. Propuestas y respuestas, México, UNAM-Centro de Investigaciones sobre América del Norte, 262 p.

VICE (2013) El purgatorio de los deportados, [en ligne] consulté le 12/11/2013. URL : http://www.vice.com/es_mx/Fringes/el-purgatorio-de-los-deportados

Wessler Seth Freed, Portillo Gonzales Esther and Apollon Dominique (2011) Shattered Families: The Perilous Intersection of Immigration Enforcement and the Child Welfare System, New York, Applied Research Center, 65 p.

Wihtol de Wenden Catherine (2012) Atlas des migrations. Un équilibre mondial à inventer, Paris, Éditions Autrement, 96 p.

Zúñiga Herrera Elena, Arroyo Alejandre Jesús, Escobar Latapí Agustín et Verduzco Igartúa Gustavo (2006) Migración Mexico-Estados Unidos: implicaciones y retos para ambos países, Guadalajara, CONAPO-UG-COLMEX-CIESASCasa Juan Pablos, 355 p. 


\section{Olga Odgers Ortiz et Amalia E. Campos Delgado}

\section{$\cdots$ Figés dans le mouvement : périodes et espaces d'attente des migrants mexicains expulsés des États-Unis}

Aujourd'hui, après un siècle marqué par une forte mobilité, le système migratoire Mexique/États-Unis s'essouffle : le contrôle des frontières a été durci et de nouvelles procédures d'expulsion des migrants en situation irrégulière ont été mises en place. Les auteurs explorent ici les contextes et les expériences de ceux qui, expulsés et dans l'impossibilité de choisir une destination, restent " figés dans le mouvement ", entre l'espoir de retrouver un territoire américain qu'ils ont fait leur et les obstacles que suppose le renforcement des mécanismes transnationaux de contrôle migratoire. Retourner aux États-Unis coûte que coûte, regagner leur lieu d'origine ou s'installer indéfiniment dans une ville frontalière constituent les choix dérivés des mécanismes d'exclusion axés sur le contrôle de la mobilité. Au binôme mobilité/immobilité s'ajoute ainsi la catégorie " en suspens ", celle de milliers de migrants forcés à retourner dans un pays où ils ne se sentent plus chez eux.

\section{Suspended in the Movement: Waiting Periods and Spaces for Mexicans Deported from the United States}

Overwhelmed by the intensity of the last century's mobility, the Mexico-USA migratory system is going through a phase of depletion, through a weakening process together with the strengthening of border control mechanisms and the establishment of new expulsion proceedings for illegal immigrants. This paper addresses the backgrounds and experiences of those deportees who get "frozen in motion", facing the impossibility to choose where they wish to live. The movement-control focused exclusion mechanisms have led to three consequences: the return no matter what to the USA, the return to original communities and the unlimited journeys within border cities. Thousands of migrants, forced to return to a country that is no longer their home, have discovered they belong to a new category: between mobility and immobility, they find themselves in an "unresolved condition".

\section{Suspendidos en el movimiento: periodos y espacios de espera de los migrantes mexicanos deportados de los Estados Unidos}

Después de un siglo de intensa movilidad, al inicio del siglo XXI el sistema migratorio México-Estados Unidos entra en una fase de agotamiento que se acompaña con el reforzamiento del control fronterizo y nuevos procesos de expulsión de migrantes indocumentados. En este artículo, los autores abordan los contextos y las experiencias de los expulsados, que quedan atrapados en el movimiento, ante la imposibilidad de elegir a dónde ir. Las decisiones que toman están marcadas por la tensión entre la expectativa de recuperar su vida en aquel país y los mecanismos transnacionales de control de la movilidad. El retorno a todo costo a Estados Unidos, el retorno a las comunidades de origen, o la permanencia en las ciudades fronterizas, son consecuencias de los mecanismos de exclusión centrados en el control de los desplazamientos. A las categorías de movilidad e inmovilidad se suma la condición "en vilo» en que actualmente viven miles de migrantes obligados a retornar a un país que ya no es su hogar. 